\title{
PARABOLIC SYSTEM OF CHEMOTAXIS: BLOWUP IN A FINITE AND THE INFINITE TIME
}

\author{
TAKASI SENBA* AND TAKASHI SUZUKI ${ }^{\dagger}$
}

1. Introduction. The purpose of the present paper is to study blowup mechanism for a system of parabolic equations. It arises in mathematical biology to describe the chemotactic feature of slime molds.

We take the form proposed by Nanjundiah [20], simplifying the one previously given by Keller and Segel [14]. It is stated as follows, where $u=u(x, t)$ and $v=v(x, t)$ stand the density of slime molds and the concentration chemical substances secreted by them, respectively:

$$
\begin{array}{rll}
u_{t}=\nabla \cdot(\nabla u-u \nabla v) & \text { in } & \Omega \times(0, T) \\
\tau v_{t}=\Delta v-a v+u & \text { in } & \Omega \times(0, T) \\
\partial u / \partial \nu=\partial v / \partial \nu=0 & \text { on } & \partial \Omega \times(0, T) \\
\left.u\right|_{t=0}=u_{0}(x) & \text { in } & \Omega \\
\left.v\right|_{t=0}=v_{0}(x) & \text { in } & \Omega
\end{array}
$$

Here, $\Omega \subset \mathbf{R}^{2}$ denotes a bounded domain with smooth boundary $\partial \Omega, \nu$ is the outer normal unit vector, and $\tau>0$ and $a>0$ are constants. The initial values $u_{0}(x)$ and $v_{0}(x)$ are smooth, non-negative, and $u_{0} \not \equiv 0$.

The first equation describes the conservation of mass; the effect of diffusion, $\nabla u$, and that of chemotaxis, $u \nabla v$, are competing for $u$ to vary. The second equation is linear and indicates that the chemical mateiral $v$ diffuses by itself, is produced by $u$, and is destroyed by the rate $a>0$. The constant $\tau>0$ is small and shows that the time scales for $u$ and $v$ are different.

Alt [2] approached the problem of modelling from the microscopic point of view. A stochastic process was introduced, and the first equation was derived from biophysical and biochemical structures of slime molds. System (1.1) is supposed to explain the process of the concentration of mass and the formation of spores of slime molds. Behavior of the solution global in time is quite important.

Unique existence, positivity, and regularity of the classical solution of (1.1) are assured locally in time by Yagi [26] and Biler [3]. Henceforth, $T_{\max }>0$ denotes the maximal time of existence for the classical solution $(u, v)$.

It is easy to see that the first component $u$ preserves $L^{1}$ norm. We have

$$
\|u(t)\|_{1}=\left\|u_{0}\right\|_{1} \equiv \lambda
$$

from the first equation. This implies also that

$$
\|v(t)\|_{1}=e^{-\frac{a}{\tau} t}\left\|v_{0}\right\|_{1}+a^{-1}\left(1-e^{-\frac{a}{\tau} t}\right) \lambda
$$

from the second equation.

The existence of Lyapunov function is to be noted. We have

$$
\frac{d}{d t} W(u, v)+\tau \int_{\Omega} v_{t}^{2} d x+\int_{\Omega} u|\nabla(\log u-v)|^{2} d x=0,
$$

*Department of Applied Mathematics, Faculty of Engineering, Miyazaki University, Japan.

† Department of Mathematics, Graduate School of Science, Osaka University, Japan. 
where

$$
W(u, v)=\int_{\Omega} u \log u d x-\int_{\Omega} u v d x+\frac{1}{2} \int_{\Omega}\left(|\nabla v|^{2}+a v^{2}\right) d x .
$$

This formula was found by Nagai, Senba, and Yoshida [18], Gajewski and Zacharias [8], and Biler [3] independently. As a consequence, they were able to show that $\lambda=\left\|u_{0}\right\|_{1}<4 \pi$ implies $T_{\max }=+\infty$ in use of a variant of the Trudinger-Moser inequality by Chang and Yang [6], and also Moser's iteration scheme (c.f. Alikakos [1]). This fact is referred to as the threshold of the initial mass.

Herrero and Velázquez [10], [11] applied the method of matched asymptotic expansions. They constructed a family of radially symmetric solutions on $\Omega=$ $\left\{x \in \mathbf{R}^{2}|| x \mid<1\right\}$, satisfying

$$
u(x, t) d x \rightarrow 8 \pi \delta_{0}(d x)+f(x) d x
$$

as $t \nearrow T_{\max }<+\infty$ in $\mathcal{M}(\bar{\Omega})$, where $f \in C(\bar{\Omega} \backslash\{0\}) \cap L^{1}(\Omega)$ is a non-negative function. This fact is referred to as the chemotactic collapse of the solution.

Those properties, threshold of the initial mass and chemotactic collapse of the solution were suspected by Childress and Percus [7]. They are regarded as the consequences of the important phenomenon of biology, formation of spores described above.

The argument of [7] is as follows. Consider the stationary problem of (1.1):

$$
\begin{array}{lll}
\nabla \cdot(\nabla U-U \nabla V)=0 & \text { in } & \Omega \\
\Delta V-a V+U=0 & \text { in } & \Omega \\
\partial U / \partial \nu=\partial V / \partial \nu=0 & \text { on } & \partial \Omega
\end{array}
$$

Writing the first equation as

$$
\nabla \cdot U \nabla(\log U-V)=0
$$

we see that $\log U-V=\log \sigma$ holds with some constant $\sigma>0$. In use of the parameter $\lambda=\|U\|_{1}$, this relation is indicated as

$$
U=\lambda e^{V} / \int_{\Omega} e^{V} d x
$$

Then the elliptic eigenvalue problem with non-local term,

$$
-\Delta V+a V=\lambda e^{V} / \int_{\Omega} e^{V} d x \quad \text { in } \quad \Omega, \quad \frac{\partial V}{\partial \nu}=0 \quad \text { on } \quad \partial \Omega
$$

arises from the second equation.

Computing numerically, they observed that only constant solutions are admitted as radially symmetric solutions on $\Omega=\left\{x \in \mathbf{R}^{2}|| x \mid<1\right\}$ of (1.4), if $\lambda \in(0,8 \pi)$. Those considerations led them to conjecture that $\lambda=\left\|u_{0}\right\|_{1}<8 \pi$ implies $T_{\max }=+\infty$ in (1.1), while $T_{\max }<+\infty$ can occur if $\lambda>8 \pi$, "because blowup solutions should have radially symmetric features around blowup points". Remember that actually it is shown that $\lambda<4 \pi$ implies $T_{\max }=+\infty$.

The threshold on $\lambda=\left\|u_{0}\right\|_{1}$ for $T_{\max }=+\infty$ is expected only when the space dimension $N$ is two. If $N=1$, we always have $T_{\max }=+\infty$. If $N=3, T_{\max }<+\infty$ can 
occur regardless of $\lambda$, and rather interesting features of the solution can be observed. See [15] and the references therein for those facts concerning other space dimensions.

First, Jäger and Luckhaus [13] approached that conjecture rigorously. For a more simplified system they showed that $\lambda=\left\|u_{0}\right\|_{1} \ll 1$ implies $T_{\max }=+\infty$, while $T_{\max }\langle+\infty$ can happen when $\lambda \gg 1$. Later Nagai [15] proved that the conjecture holds in the affirmative for radially symmetric solutions of

$$
\begin{array}{rll}
u_{t}=\nabla \cdot(\nabla u-u \nabla v) & \text { in } & \Omega \times(0, T) \\
0=\Delta v-a v+u & \text { in } & \Omega \times(0, T) \\
\partial u / \partial \nu=\partial v / \partial \nu=0 & \text { on } & \partial \Omega \times(0, T) \\
\left.u\right|_{t=0}=u_{0}(x) & \text { in } & \Omega .
\end{array}
$$

System (1.5) is the limiting case of (1.1) as $\tau \searrow 0$ and obeys a similar features to the one introduced by [13]. In this situation, $\lambda=\left\|u_{0}\right\|_{1}<8 \pi$ implies $T_{\max }=+\infty$, while $T_{\max }<+\infty$ can occur if $\lambda>8 \pi$. However, the discrepancy between $8 \pi$ and $4 \pi$ in radial and non-radial cases is essential as the authors have clarified in [21], [25], and $[22]$.

We began the study by re-examining the stationary problem ([21]). First observation is that problem (1.4) has a variational structure; the solution is characterized as a critical point of the functional

$$
J_{\lambda}(v)=\frac{1}{2} \int_{\Omega}\left(|\nabla v|^{2}+a v^{2}\right) d x-\lambda \log \left(\frac{1}{|\Omega|} \int_{\Omega} e^{v} d x\right)
$$

of $v \in H^{1}(\Omega)$. This implies that the linearized operator around a stationary solution $V$ is realized as a self-adjoint one in $L^{2}(\Omega)$ associated with the bilinear form

$$
\mathcal{A}(\phi, \phi)=\int_{\Omega}\left(|\nabla \phi|^{2}+a \phi^{2}-p \phi^{2}\right) d x+\frac{1}{\lambda}\left(\int_{\Omega} p \phi d x\right)^{2}
$$

of $\phi \in H^{1}(\Omega)$, where $p=\lambda e^{V} / \int_{\Omega} e^{V} d x$. In particular, the linearized stability of $V$ is introduced in this sense. We also noticed that the methods developed in our former works on Dirichlet problem are still valid for this case.

Among others are the application of the complex function theory to the blowup analysis of the family of solutions ([19]), and the use of the rearrangement technique relative to a round sphere for spectral analysis of the linearized operator $([24])$. Consequently, we found that the set of stationary solutions $\mathcal{C}=\{(\lambda, V)\}$ of $(1.4)$ is much richer than the suspected, and some members are taking significant roles in the nonstationary problem. Many suggestions were obtained such as the behaviors global in time, the blowup mechanism, the dynamics, and so forth.

For instance, as is expected from the numerical computation, it is actually proven that if $\Omega=\left\{x \in \mathbf{R}^{2}|| x \mid<1\right\}$ and $\lambda \in(0,8 \pi)$, each radially symmetric stationary solution is a constant. On the contrary, there is a family of non-radial solutions bifurcation from constant solutions in this case. It is absorbed into the hyperplane $\lambda=4 \pi$ with the singular limit having one singular point on the boundary up to the rotation of $x$ around the origin. That bifurcation occurs in $\lambda<4 \pi$ if $0<a \ll 1$ and the bifurcated solutions are linearized stable. Also it is shown that any solution is linearized unstable if $0<\lambda-4 \pi \ll 1$. We suspected that only some constant is admitted as a stationary solution for $0<a \ll 1$ and $\lambda \in(4 \pi, 8 \pi)$.

Those observations to the stationary problem led us to conjecture that the mass of generic non-stationary solutions concentrates mostly to a point on the boundary 
as $t \rightarrow+\infty$ if $0<4 \pi-\lambda \ll 1$ and $0<a \ll 1$, where $\lambda=\left\|u_{0}\right\|_{1}$. Furthermore, the blowup solution of (1.1) should have only one blowup point on the boundary if $\lambda=\left\|u_{0}\right\|_{1} \in(4 \pi, 8 \pi)$ even in the general case. We suspected that "a half spore" will be created on the boundary in this case.

This conjecture, based on a heuristic argument, was supported by [25] from the viewpoint of dynamical systems; any linearized stable stationary solution $V(x)$ of $J_{\lambda}$ is dynamically stable in (1.1). More precisely, if $V(x)$ is a strict local minimum of $J_{\lambda}$, then the conditions

$$
\left\|u_{0}\right\|_{1}=\lambda, \quad\left\|u_{0}-U\right\|_{L \log L} \ll 1, \quad \text { and } \quad\left\|v_{0}-V\right\|_{H^{1}} \ll 1
$$

imply $T_{\max }=+\infty$ and $\lim _{t \rightarrow \infty}\|u(t)-U\|_{\infty}=\lim _{t \rightarrow \infty}\|v(t)-V\|_{\infty}=0$ in (1.1), where $U=$ $\lambda e^{V} / \int_{\Omega} e^{V} d x$ and $\|\cdot\|_{L \log L}$ denotes the Zygmund norm.

Key structures for the proof are the following. First, each term of the Lyapunov function $W(u, v)$ is regarded as a variant of Zygmund norm of $u$, the paring between $u$ and $v$, and the $H^{1}$ norm of $v$, respectively. Next, there are local isomorphism between the Zygmund space $L \log L$ and the Hardy space $\mathcal{H}^{1}$, paring between $\mathcal{H}^{1}$ and BMO, and imbedding $H^{1} \subset$ BMO. Of course, the inequality

$$
W(u(t), v(t)) \leq W\left(u_{0}, v_{0}\right) \quad\left(t \in\left[0, T_{\max }\right)\right)
$$

is made use of. Another observation is that $W$ and $J_{\lambda}$ are so related as

$$
W\left(\frac{\lambda e^{v}}{\int_{\Omega} e^{v} d x}, v\right)=J_{\lambda}(v)+\lambda \log (\lambda|\Omega|) .
$$

See the original paper [25] for more details.

The blowup mechanism of (1.5) is now well understood as is expected by [22]. If $T_{\max }<+\infty$ the blowup set of $u$,

$$
\begin{aligned}
\mathcal{B}= & \left\{x \in \bar{\Omega} \mid \text { there exists } x_{k} \rightarrow x \text { and } t_{k} \nearrow T_{\max }\right. \\
& \text { satisfying } \left.u\left(x_{k}, t_{k}\right) \rightarrow+\infty\right\}
\end{aligned}
$$

is finite. More precisely, we have

$$
\sharp(\mathcal{B} \cap \partial \Omega)+2 \sharp(\mathcal{B} \cap \Omega) \leq\left\|u_{0}\right\|_{1} /(4 \pi) .
$$

Furthermore, there exist a mapping $m: \mathcal{B} \rightarrow[4 \pi, \infty)$ with $\left.m\right|_{\mathcal{B} \cap \Omega} \geq 8 \pi$ and a nonnegative function $f \in C(\bar{\Omega} \backslash \mathcal{B}) \cap L^{1}(\Omega)$ satisfying

$$
u(x, t) d x \rightarrow \sum_{x_{0} \in \mathcal{B}} m\left(x_{0}\right) \delta_{x_{0}}(d x)+f(x) d x
$$

in $\mathcal{M}(\bar{\Omega})$ as $t \nearrow T_{\max }$. Delicate analysis is made on many places, but a cancellation scheme of the singularity in a reduced integral equation is a key structure. Then, some local behaviors of the Green's function are made use of.

The case $\tau>0$ is more difficult. Profile of the chemotactic collapse (1.6) is proven when the Lyapunov function $W$ is bounded, or $u$ and $v$ are radially symmetric, or $\lambda=4 \pi$ ([17], [9]). Right now we expect infinite blowup sets for other cases.

Another question is the possibility of $m\left(x_{0}\right)>8 \pi$ for $x_{0} \in \mathcal{B} \cap \Omega$, or $m\left(x_{0}\right)>4 \pi$ for $x_{0} \in \mathcal{B} \cap \partial \Omega$ in (1.6). It will be studied in a forthcoming paper of us. 
So far, sufficient conditions for $T_{\max }<+\infty$ have been given mostly for (1.5). In the present paper, we refine the condition of [16] concerning the boundary blowup of the solution in a finite time. Another aim is to give an alternative proof of a theorem by Horstmann and Wang [12]. It is concerned with the blowup (possibly in the infinite time) of the solution of (1.1). We believe that the argument presented here is more detailed. Applying it to (1.5), we shall show that $\mathcal{B}=\{p\}$ and $m(p)=8 \pi$ occur, if $T_{\max }=+\infty, u$ and $v$ are radially symmetric, and $p$ is the center of $\Omega$.

Our theorems are stated as follows.

First, in [21], it is shown that if $V_{\lambda}$ 's are solutions to (1.4), $\lambda \rightarrow \lambda_{0} \in[0, \infty)$, and $\left\|V_{\lambda}\right\|_{\infty} \rightarrow+\infty$, then $\lambda_{0} \in 4 \pi \mathbf{N}$. The number of blowup points of this family satisfies

$$
\sharp(\mathcal{B} \cap \partial \Omega)+2 \sharp(\mathcal{B} \cap \Omega)=\frac{\lambda_{0}}{4 \pi} .
$$

We have

$$
\underline{j}_{\lambda} \equiv\left\{J_{\lambda}(v) \mid v \text { solves }(1.4)\right\}>-\infty
$$

if $\lambda \in(0, \infty) \backslash 4 \pi \mathbf{N}$. The following theorem shows that the blowup of the nonstationary solution occurs in a finite or the infinite time if $u_{0}$ and $v_{0}$ satisfy

$$
\left\|u_{0}\right\|_{1}=\lambda \quad \text { and } \quad W\left(u_{0}, v_{0}\right)<\underline{j}_{\lambda}+\lambda \log (\lambda|\Omega|) .
$$

It is nothing but the one proven by Horstmann and Wang [12], but we shall provide different arguments here.

THEOREM 1. If (1.7) holds, then the solution of (1.1) satisfies

$$
\lim _{t \nearrow T_{\max }}\|u(t)\|_{\infty}=+\infty
$$

More precisely, we have

$$
\begin{aligned}
& \lim _{t \nearrow T_{\max }} \int_{\Omega} u \log u d x=\lim _{t \nearrow T_{\max }} \int_{\Omega} u v d x \\
& \quad=\lim _{t \nearrow T_{\max }} \int_{\Omega}|\nabla v|^{2} d x=\lim _{t \nearrow T_{\max }} \int_{\Omega} e^{\alpha v} d x=+\infty
\end{aligned}
$$

for any $\alpha>1$. Here the case $T_{\max }=+\infty$ is admitted.

If $\Omega=\left\{x \in \mathbf{R}^{2}|| x \mid<1\right\}$, we have

$$
\underline{j}_{\text {rad, } \lambda} \equiv\left\{J_{\lambda}(v) \mid v \text { is a radial solution of }(1.4)\right\}>-\infty
$$

for $\lambda \in(0, \infty) \backslash\{8 \pi\}$. We can find a radial function $u_{0}$ satisfying

$$
\left\|u_{0}\right\|_{1}=\lambda \quad \text { and } \quad W\left(u_{0}, v_{0}\right)<\underline{j}_{r a d, \lambda}+\lambda \log (\lambda|\Omega|)
$$

for $v_{0}=(-\Delta+a)^{-1} u_{0}$ similarly. Then, (1.8) or (1.9) holds to the solution $u$ of (1.5). In use of the argument presented in this paper for the previous theorem, we can show the following fact. 
THEOREM 2. Let $u_{0}$ be radially symmetric and satisfy (1.10). Then, the solution $u$ of (1.5) satisfies

$$
\lim _{R \searrow 0} \liminf _{t \nearrow T_{\max }} \int_{\{|x|<R\}} u d x=\lim _{R \searrow 0} \limsup _{t \nearrow T_{\max }} \int_{\{|x|<R\}} u d x=8 \pi
$$

if $T_{\max }=+\infty$.

Note that if $T_{\max }<+\infty$ and $u_{0}$ is radially symmetric, then $\sharp \mathcal{B}=1$ and furthermore (1.6) holds with $x_{0}=0$ for the solution $u$ of (1.5). In both cases of $T_{\max }=+\infty$ and $T_{\max }<+\infty$, the solution develops a singularity like a delta function at the origin.

The last theorem gives a criterion for the boudary blowup of the solution $u$ of (1.5) in a finite time. It is a refinement of the result obtained by [16]. Suppose that $\partial \Omega$ is smooth at $x_{0} \in \partial \Omega$ so that there exists a conformal mapping sending the intersection of $\partial \Omega$ and a neighborhood of $x_{0}$ into the real axis.

THEOREM 3. There exists $\eta>0$ such that

$$
\int_{\Omega \cap B_{R}\left(x_{0}\right)} u_{0}(x) d x>4 \pi
$$

and

$$
\frac{1}{R^{2}} \int_{\Omega \cap B_{R}\left(x_{0}\right)} u_{0}(x)\left|x-x_{0}\right|^{2} d x<\eta
$$

imply $T_{\max }<+\infty$ for the solution $u$ of (1.5), where

$$
B_{R}\left(x_{0}\right)=\left\{x \in \mathbf{R}^{2}|| x-x_{0} \mid<R\right\}
$$

for $R>0$.

Precisely, $\eta$ is determined by $\lambda=\left\|u_{0}\right\|_{1}$ and $\left\|u_{0}\right\|_{L^{1}\left(\Omega \cap B_{R}\left(x_{0}\right)\right)}$. Note that if $\lambda \in(4 \pi, 8 \pi)$, there exists exactly one blowup point on $\partial \Omega$.

In proving Theorem 2, we make use of the arguments for the proof of Theorems 1 and 3 . Theorems 1,2 , and 3 are proven in sections 2,4 , and 3, respectively.

2. A blowup criterion. This section is devoted to the proof of Theorem 1 . We study (1.1) for the general domain, taking $a=1$ and $\tau=1$ for simplicity.

In the previous work [23], the authors proved (1.9) for the case of $T_{\max }<+\infty$. The argument developed there is valid even for the case of $T_{\max }=+\infty$ if

$$
\lim _{t \nearrow+\infty} W(u(t), v(t))=-\infty
$$

is satisfied. We have only to show (1.9) for the other case,

$$
T_{\max }=+\infty \quad \text { and } \quad \lim _{t \nearrow+\infty} W(u(t), v(t))>-\infty .
$$

Actually, relation (1.8) follows from (1.9).

We shall show that (2.1) and (1.7) imply

$$
\lim _{t \nearrow+\infty} \int_{\Omega} u \log u d x=+\infty
$$


Becaue of

$$
\int_{\Omega} u \log u d x \leq \int_{\Omega} u v d x+W(u, v)
$$

then $\lim _{t \nearrow+\infty} \int_{\Omega} u v d x=+\infty$ follows. In use of Young's inequality we have

$$
\begin{aligned}
\alpha \int_{\Omega} u v d x & \leq \int_{\Omega} u \log u d x+e^{-1} \int_{\Omega} e^{\alpha v} d x \\
& \leq \int_{\Omega} u v d x+W(u, v)+e^{-1} \int_{\Omega} e^{\alpha v} d x
\end{aligned}
$$

and hence $\lim _{t \nearrow+\infty} \int_{\Omega} e^{\alpha v(x, t)} d x=+\infty$ holds for $\alpha>1$. This implies

$$
\lim _{t \nearrow+\infty} \int_{\Omega}|\nabla v(t)|^{2} d x=+\infty
$$

by Chang-Yang's inequality (see [23]). The proof will be complete in this way.

Suppose the contrary: $\liminf _{t \nearrow+\infty} \int_{\Omega} u \log u d x<+\infty$. There exist a constant $C_{*}>0$ and a sequence $t_{k} \nearrow+\infty$ satisfying

$$
\int_{\Omega} u\left(t_{k}\right) \log u\left(t_{k}\right) d x \leq C_{*}
$$

Assumption (2.1) now gives

$$
\int_{0}^{\infty} \int_{\Omega}\left(v_{t}^{2}+u|\nabla(\log u-v)|^{2}\right) d x d t<+\infty
$$

Letting $k \gg 1$, we may suppose that

$$
\int_{t_{k}}^{\infty} \int_{\Omega} v_{t}^{2} d x d t<1
$$

In [23], the inequality

$$
\begin{aligned}
& \frac{d}{d t} \int_{\Omega} u \log u d x \leq 2 K^{2}\left\|u_{0}\right\|_{1}^{2}+\frac{1}{4} \int_{\Omega} v_{t}^{2} d x \\
& \quad+4|\Omega| \exp \left(4 K^{2} \int_{\Omega} u \log u d x+4 K^{2} e^{-1}|\Omega|\right)
\end{aligned}
$$

is shown with a constant $K>0$ determined by $\Omega$. Take $\delta_{*}>0$ satisfying

$$
\delta_{*}\left\{2 K^{2}\left\|u_{0}\right\|_{1}^{2}+4|\Omega| \exp \left(4 K^{2}\left(C_{*}+1\right)+4 K^{2} e^{-1}|\Omega|\right)\right\}=\frac{1}{4} .
$$

For some $\tilde{t}_{k} \in\left(t_{k}, t_{k}+\delta_{*}\right)$ we have

$$
\int_{\Omega} u(t) \log u(t) d x<C_{*}+1 \quad\left(t_{k} \leq t<\tilde{t}_{k}\right) .
$$


Then inequality (2.6) implies

$$
\begin{aligned}
& \int_{\Omega} u\left(\tilde{t}_{k}\right) \log u\left(\tilde{t}_{k}\right) d x \leq \frac{1}{4} \int_{t_{k}}^{\infty} \int_{\Omega} v_{t}^{2} d x d t+\int_{\Omega} u\left(t_{k}\right) \log u\left(t_{k}\right) d x \\
& +\left(2 K^{2}\left\|u_{0}\right\|_{1}^{2}+4|\Omega| \exp \left(4 K^{2}\left(C_{*}+1\right)+4 K^{2} e^{-1}|\Omega|\right)\right)\left(\tilde{t}_{k}-t_{k}\right) \\
& \quad \leq C_{*}+\frac{1}{2} .
\end{aligned}
$$

Because $t \in\left[t_{k}, t_{k}+\delta_{*}\right] \mapsto \int_{\Omega} u(t) \log u(t) d x$ is continuous, this means that

$$
\int_{\Omega} u(t) \log u(t) d x \leq C_{*}+1 \quad\left(t_{k} \leq t \leq t_{k}+\delta_{*}\right) .
$$

Here, $\delta_{*}>0$ is independent of $k$. We have

$$
\begin{aligned}
& \lim _{k \rightarrow \infty} \inf _{t \in\left(t_{k}, t_{k}+\delta_{*}\right)} \int_{\Omega}\left(v_{t}^{2}(t)+u(t)|\nabla(\log u(t)-v(t))|^{2}\right) d x \\
& \leq \delta_{*}^{-1} \lim _{k \rightarrow \infty} \int_{t_{k}}^{t_{k}+\delta_{*}} \int_{\Omega}\left(v_{t}^{2}(t)+u(t)|\nabla(\log u(t)-v(t))|^{2}\right) d x d t \\
& =0
\end{aligned}
$$

by (2.5). With some $\hat{t}_{k} \in\left[t_{k}, t_{k}+\delta_{*}\right]$ it holds that

$$
\lim _{k \rightarrow \infty} \int_{\Omega}\left(v_{t}^{2}\left(\hat{t}_{k}\right)+u\left(\hat{t}_{k}\right)\left|\nabla\left(\log u\left(\hat{t}_{k}\right)-v\left(\hat{t}_{k}\right)\right)\right|^{2}\right) d x=0 .
$$

We have

$$
u|\nabla(\log u-v)|^{2}=4 e^{v}\left|\nabla\left(u e^{-v}\right)^{\frac{1}{2}}\right|^{2} \geq 4\left|\nabla\left(u e^{-v}\right)^{\frac{1}{2}}\right|^{2}
$$

and hence

$$
\lim _{k \rightarrow \infty}\left\|\nabla\left(u\left(\hat{t}_{k}\right) e^{-v\left(\hat{t}_{k}\right)}\right)^{1 / 2}\right\|_{2}=0
$$

follows.

On the other hand we have $\int_{\Omega} u e^{-v} d x \leq\|u\|_{1}=\lambda$. Passing through a subsequence, we get

$$
\lim _{k \rightarrow \infty} \frac{1}{|\Omega|} \int_{\Omega}\left(u\left(\hat{t}_{k}\right) e^{-v\left(\hat{t}_{k}\right)}\right)^{1 / 2} d x=C_{0}
$$

with a constant $C_{0} \geq 0$. Therefore, Poincaré-Wirtinger's inequality implies $\left(u\left(\hat{t}_{k}\right) e^{-v\left(\hat{t}_{k}\right)}\right)^{1 / 2} \rightarrow C_{0}$ in $H^{1}(\Omega)$, and hence we have

$$
u\left(\hat{t}_{k}\right) e^{-v\left(\hat{t}_{k}\right)} \rightarrow C_{0}^{2} \quad \text { in } \quad L^{p}(\Omega)
$$

for any $p>1$. 
Relation (2.8) gives

$$
\lim _{k \rightarrow \infty}\left\|v_{t}\left(\hat{t}_{k}\right)\right\|_{2}=0
$$

Noting $\|u(t)\|_{1}=\lambda$, we apply an inequality of Brezis and Merle [4] to the second equation of (1.1). With some $\alpha>0$ it holds that

$$
\sup _{k} \int_{\Omega} e^{\alpha v\left(\hat{t}_{k}\right)} d x<+\infty .
$$

Inequalities (2.4) and (2.7) imply

$$
\sup _{k} \int_{\Omega} u\left(\hat{t}_{k}\right) v\left(\hat{t}_{k}\right) d x<+\infty .
$$

The second equation of (1.1) gives that

$$
\|\nabla v\|_{2}^{2}+\|v\|_{2}^{2}=\int_{\Omega} u v d x-\int_{\Omega} v_{t} v d x \leq \int_{\Omega} u v d x+\frac{1}{2}\left\|v_{t}\right\|_{2}^{2}+\frac{1}{2}\|v\|_{2}^{2} .
$$

Relation (2.10) now implies

$$
\sup _{k}\left\|v\left(\hat{t}_{k}\right)\right\|_{H^{1}(\Omega)}<+\infty .
$$

Passing through a subsequence, we have

$$
v\left(\hat{t}_{k}\right) \rightarrow v_{\infty} \quad \text { in } \quad H^{1}(\Omega) \quad \text { and } \quad e^{v\left(\hat{t}_{k}\right)} \rightarrow e^{v_{\infty}} \quad \text { in } \quad L^{p}(\Omega)
$$

with some $v_{\infty} \in H^{1}(\Omega)$ for $p>1$. The latter convergence is a consequence of the compact imbedding $H^{1}(\Omega) \subset L^{p}(\Omega)$ and Chang-Yang's inequality and details are left to the reader.

We set $t=\hat{t}_{k}$ and make $k \rightarrow \infty$ in the second equation of (1.1). Relations (2.9) and (2.11) imply

$$
u\left(\hat{t}_{k}\right)=\left(u\left(\hat{t}_{k}\right) e^{-v\left(\hat{t}_{k}\right)}\right) \cdot e^{v\left(\hat{t}_{k}\right)} \rightarrow C_{0}^{2} e^{v_{\infty}} \quad \text { in } \quad L^{p}(\Omega)
$$

for $p>1$. In use of (2.10) and (2.11), we get

$$
-\Delta v_{\infty}+v_{\infty}=C_{0}^{2} e^{v_{\infty}} \quad \text { in } \quad \Omega, \quad \frac{\partial v_{\infty}}{\partial \nu}=0 \quad \text { on } \quad \partial \Omega .
$$

Furthermore, equality (1.2) gives $\left\|v_{\infty}\right\|_{1}=\lambda$ and hence $C_{0}^{2}=\lambda / \int_{\Omega} e^{v_{\infty}} d x$.

Letting $u_{\infty}=\lambda e^{v_{\infty}} / \int_{\Omega} e^{v_{\infty}} d x$, we have $u\left(\hat{t}_{k}\right) \rightarrow u_{\infty}$ in $L^{p}(\Omega)$. This implies

$$
\lim _{k \rightarrow \infty} \int_{\Omega} u\left(\hat{t}_{k}\right) \log u\left(\hat{t}_{k}\right) d x=\int_{\Omega} u_{\infty} \log u_{\infty} d x
$$

We also have

$$
\lim _{k \rightarrow \infty} \int_{\Omega} u\left(\hat{t}_{k}\right) v\left(\hat{t}_{k}\right) d x=\int_{\Omega} u_{\infty} v_{\infty} d x
$$


and

$$
\liminf _{k \rightarrow \infty} \int_{\Omega}\left(\left|\nabla v\left(\hat{t}_{k}\right)\right|^{2}+v\left(\hat{t}_{k}\right)^{2}\right) d x \geq \int_{\Omega}\left(\left|\nabla v_{\infty}\right|^{2}+v_{\infty}^{2}\right) d x .
$$

Those relations contradict assumption (1.7) as

$$
\begin{gathered}
W\left(u_{0}, v_{0}\right) \geq \lim _{k \rightarrow \infty} W\left(u\left(\hat{t}_{k}\right), v\left(\hat{t}_{k}\right)\right) \geq W\left(u_{\infty}, v_{\infty}\right) \\
=J_{\lambda}\left(v_{\infty}\right)+\lambda \log (\lambda|\Omega|) \geq j_{\lambda}+\lambda \log (\lambda|\Omega|) .
\end{gathered}
$$

The proof is complete.

For solutions of (1.5), inequality (2.3) is improved as

$$
\int_{\Omega} u \log u d x \leq \frac{1}{2} \int_{\Omega} u v d x+W(u, v) .
$$

This fact implies that $\alpha$ can be taken as $\alpha>\frac{1}{2}$ in (1.9) in the case of (1.5). Except for this improvement, the results stated in Theorem 1 are still valid for solutions of (1.5). A related result is shown in [9] for solutions of (1.1).

3. Boundary blowup of the solution. This section is devoted to the proof of Therem 3. We study (1.5) on the general domain $\Omega$ with $\partial \Omega$ sufficiently smooth around $x_{0} \in \partial \Omega$, and $u$ denotes the solution. Theorem 3 is proven by localizing the argument of [15].

There exists a conformal mapping $X=\left(X_{1}, X_{2}\right)$ defined on $\Omega \cap B_{R}\left(x_{0}\right)$ satisfying $X: \Omega \cap B_{R}\left(x_{0}\right) \rightarrow \mathbf{R}_{+}^{2} \equiv\left\{\left(x_{1}, x_{2}\right) \in \mathbf{R}^{2} \mid x_{2}>0\right\}$ and $X\left(\partial \Omega \cap B_{R}\left(x_{0}\right)\right) \subset \partial \mathbf{R}_{+}^{2}$. We have $\left(\partial X_{1}\right) /(\partial \nu)=0$ on $\partial \Omega$. Without loss of generality, we can assume $x_{0}=0$, $\nu\left(x_{0}\right)=(0,-1)$, and

$$
\frac{\partial X}{\partial x}\left(x_{0}\right)=\mathrm{id}
$$

Let $\phi$ be a smooth function defined on $\bar{\Omega}$ satisfying the homogeneous Neumann boundary condition. In [22], it is shown that

$$
\left|\frac{d}{d t} \int_{\Omega} u \phi d x\right| \leq\|\Delta \phi\|_{\infty}\left\|u_{0}\right\|_{1}+\frac{1}{2}\|\hat{\rho}\|_{L^{\infty}(\Omega \times \Omega)}\left\|u_{0}\right\|_{1}^{2}
$$

holds, where $\hat{\rho}(x, y)=\nabla \phi(x) \cdot \nabla_{x} G(x, y)+\nabla \phi(y) \cdot \nabla_{y} G(x, y)$ with $G=G(x, y)$ being the Green's function for $-\triangle+1$ with the homogeneous Neumann boundary condition. The following lemma is a consequence of Lemma 6 of [22].

LEMMA 3.1. Letting

$$
\lambda_{\phi}(t)=\int_{\Omega} u(x, t) \phi(x) d x
$$

we have

$$
\left|\frac{d}{d t} \lambda_{\phi}\right| \leq L\|\phi\|_{C^{2}(\Omega)}\left(\lambda^{2}+\lambda\right) \quad\left(t \in\left[0, T_{\max }\right)\right)
$$

with a constant $L>0$ determined by $\Omega$. 
Given $R>0$ sufficiently small, we take smooth functions $\phi_{i}(i=1,2)$ defined on $\mathbf{R}^{2}$ satisfying $0 \leq \phi_{i} \leq 1$

$$
\phi_{i}(x)= \begin{cases}1 & \left(x \in B_{4^{i} R}(0)\right) \\ 0 & \left(x \notin B_{2 \cdot 4^{i} R}(0)\right)\end{cases}
$$

and $\partial \phi_{i} / \partial \nu=0$ on $\partial \Omega$. Letting $\psi_{i}=\phi_{i}^{4}$ and $m(x)=|X(x)|^{2} / 2$, we can show the following.

LEMMA 3.2. We have

$$
\begin{aligned}
& \left|\rho(x, y)-\frac{1}{\pi} \psi_{1}(x) \psi_{2}(y)\right| \\
& \quad \leq C R^{-1}(|x|+|y|) \psi_{1}^{1 / 2}(x) \psi_{2}(y)+C R^{-1}|y| \psi_{2}(y)
\end{aligned}
$$

for

$$
\rho(x, y)=\left[\nabla\left(m \psi_{1}\right)(x) \cdot \nabla_{x} G(x, y)\right] \psi_{2}(y)+\left[\nabla\left(m \psi_{1}\right)(y) \cdot \nabla_{y} G(x, y)\right] \psi_{2}(x)
$$

with a constant $C>0$ independent of $R$.

Proof. We set $\left(x_{1}, x_{2}\right)^{*}=\left(x_{1},-x_{2}\right)$. From the proof of Lemma 6 of [22], we have

$$
G(x, y)=\frac{1}{2 \pi} \log \frac{1}{|X(x)-X(y)|}+\frac{1}{2 \pi} \log \frac{1}{\left|X(x)-X(y)^{*}\right|}+K(x, y)
$$

for $x, y \in \overline{B_{32 R}(0) \cap \Omega}$ with $K \in C^{1, \theta}\left(\overline{B_{32 R}(0) \cap \Omega} \times \overline{B_{32 R}(0) \cap \Omega}\right)$ and $\theta \in(0,1)$.

First, we take the term associated with

$$
G_{1}(x, y)=e_{1}(\xi, \eta)=\frac{1}{2 \pi} \log \frac{1}{|\xi-\eta|},
$$

where $\xi=X(x)$ and $\eta=X(y)$. Because $X$ is conformal, it holds that

$$
\left(\frac{\partial X}{\partial x}\right) \cdot\left(\frac{\partial X}{\partial x}\right)=\left|\frac{\partial X}{\partial x}\right| \cdot \mathrm{id}
$$

Letting $c(\xi)=\left|\frac{\partial X}{\partial x}\right|$ and $\Psi_{i}(\xi)=\psi_{i}(x)$, we have

$$
\begin{aligned}
& \rho_{1}(x, y) \equiv \psi_{2}(y) \nabla_{x}\left(m \psi_{1}\right)(x) \cdot \nabla_{x} G_{1}(x, y) \\
&+\psi_{2}(x) \nabla_{y}\left(m \psi_{1}\right)(y) \cdot \nabla_{y} G_{1}(x, y) \\
&=\frac{1}{2} c(\xi) \Psi_{2}(\eta) \nabla_{\xi}\left(|\xi|^{2} \Psi_{1}(\xi)\right) \cdot \nabla_{\xi} e_{1}(\xi, \eta) \\
& \quad+\frac{1}{2} c(\eta) \Psi_{2}(\xi) \nabla_{\eta}\left(|\eta|^{2} \Psi_{1}(\eta)\right) \cdot \nabla_{\eta} e_{1}(\xi, \eta) \\
&=\frac{(\xi-\eta)}{4 \pi|\xi-\eta|^{2}} \cdot\left\{c(\xi) \Psi_{2}(\eta)\left(2 \xi \Psi_{1}(\xi)+|\xi|^{2} \nabla_{\xi} \Psi_{1}(\xi)\right)\right. \\
&\left.-c(\eta) \Psi_{2}(\xi)\left(2 \eta \Psi_{1}(\eta)+|\eta|^{2} \nabla_{\eta} \Psi_{1}(\eta)\right)\right\}
\end{aligned}
$$

This implies $\rho_{1}=I+I I+I I I+I V+V$ with

$$
I=\frac{1}{2 \pi} c(\xi) \Psi_{1}(\xi) \Psi_{2}(\eta)
$$




$$
\begin{aligned}
I I & =\frac{(\xi-\eta) \cdot \eta}{2 \pi|\xi-\eta|^{2}}\left\{c(\xi) \Psi_{2}(\eta) \Psi_{1}(\xi)-c(\eta) \Psi_{2}(\xi) \Psi_{1}(\eta)\right\} \\
I I I & =\frac{(\xi-\eta)}{2 \pi|\xi-\eta|^{2}} \cdot \nabla_{\xi} \Psi_{1}(\xi) c(\xi) \Psi_{2}(\eta)\left(|\xi|^{2}-|\eta|^{2}\right) \\
I V & =\frac{(\xi-\eta)}{4 \pi|\xi-\eta|^{2}} \cdot\left(\nabla_{\xi} \Psi_{1}(\xi)-\nabla_{\eta} \Psi_{1}(\eta)\right) c(\xi) \Psi_{2}(\eta)|\eta|^{2} \\
V & =\frac{(\xi-\eta)}{4 \pi|\xi-\eta|^{2}} \cdot \nabla_{\eta} \Psi_{1}(\eta)\left(c(\xi) \Psi_{2}(\eta)-c(\eta) \Psi_{2}(\xi)\right)|\eta|^{2} .
\end{aligned}
$$

In use of (3.1), we get $c(\xi)=1+O(|x|)$ and hence

$$
I=\frac{1}{2 \pi}(1+O(|x|)) \psi_{1}(x) \psi_{2}(y)
$$

follows. Similarly, we obtain

$$
\begin{aligned}
I I= & \frac{(\xi-\eta) \cdot \eta}{2 \pi|\xi-\eta|^{2}}\left\{(c(\xi)-c(\eta)) \Psi_{2}(\eta) \Psi_{1}(\xi)\right. \\
& \left.+c(\eta) \Psi_{2}(\eta)\left(\Psi_{1}(\xi)-\Psi_{1}(\eta)\right)+c(\eta)\left(\Psi_{2}(\eta)-\Psi_{2}(\xi)\right) \Psi_{1}(\eta)\right\} \\
= & O(|\eta|) \Psi_{2}(\eta) \Psi_{1}(\xi)+O(|\eta|) O\left(R^{-1}\right) \Psi_{2}(\eta)+O(|\eta|) O\left(R^{-1}\right) \Psi_{1}(\eta) \\
= & O(|y|)\left(\psi_{2}(y) \psi_{1}(x)+O\left(R^{-1}\right) \psi_{2}(y)+O\left(R^{-1}\right) \psi_{1}(y)\right) \\
I I I= & \frac{(\xi-\eta)}{2 \pi|\xi-\eta|^{2}} \cdot \nabla_{\xi} \Psi_{1}(\xi) c(\xi) \Psi_{2}(\eta)(\xi-\eta) \cdot(\xi+\eta) \\
= & O(|\xi|+|\eta|) O\left(\nabla_{\xi} \Psi_{1}(\xi)\right) c(\xi) \Psi_{2}(\eta) \\
= & O(|x|+|y|) O\left(R^{-1}\right) \psi_{1}(x)^{1 / 2} \psi_{2}(y) \\
I V= & O\left(|\eta|^{2}\right) O\left(R^{-2}\right) \Psi_{2}(\eta)=O(|y|) O\left(R^{-1}\right) \psi_{2}(y) \\
V= & O\left(|\eta|^{2}\right) O\left(R^{-1}\right) O\left(\nabla_{\eta} \Psi_{1}(\eta)\right)=O(|y|) O\left(R^{-1}\right) \psi_{1}(y)^{1 / 2}
\end{aligned}
$$

We get

$$
\left|\rho_{1}(x, y)-\frac{1}{2 \pi} \psi_{1}(x) \psi_{2}(y)\right| \leq \frac{C}{R}(|x|+|y|) \psi_{1}(x)^{1 / 2} \psi_{2}(y)+\frac{C}{R}|y| \psi_{2}(y)
$$

by $\psi_{2} \geq \psi_{1}^{1 / 2}$.

We turn to the term associated with

$$
G_{2}(x, y)=e_{2}(\xi, \eta)=\frac{1}{2 \pi} \log \frac{1}{\left|\xi-\eta^{*}\right|} .
$$

Because of $\partial \psi_{i} / \partial \nu=0$ on $\partial \Omega$, we get

$$
\left.\frac{\partial \Psi_{i}}{\partial \xi_{2}}\right|_{\xi_{2}=0}=0
$$

for $i=1,2$. We obtain

$$
\begin{aligned}
\rho_{2}(x, y) \equiv & \psi_{2}(y) \nabla_{x}\left(m \psi_{1}\right)(x) \cdot \nabla_{x} G_{2}(x, y)+\psi_{2}(x) \nabla_{x}\left(m \psi_{1}\right)(y) \cdot \nabla_{y} G_{2}(x, y) \\
= & \frac{1}{2} c(\xi) \Psi_{2}(\eta) \nabla_{\xi}\left(|\xi|^{2} \Psi_{1}(\xi)\right) \cdot \nabla_{\xi} e_{2}(\xi, \eta) \\
& \quad+\frac{1}{2} c(\eta) \Psi_{2}(\xi) \nabla_{\eta}\left(|\eta|^{2} \Psi_{1}(\eta)\right) \cdot \nabla_{\eta} e_{2}(\xi, \eta) \\
= & V I+V I I+V I I I+I X
\end{aligned}
$$


with

$$
\begin{aligned}
V I & =\frac{\left(\xi_{1}-\eta_{1}\right)}{2 \pi\left|\xi-\eta^{*}\right|^{2}}\left\{c(\xi) \xi_{1} \Psi_{1}(\xi) \Psi_{2}(\eta)-c(\eta) \eta_{1} \Psi_{1}(\eta) \Psi_{2}(\xi)\right\} \\
V I I & =\frac{\left(\xi_{1}-\eta_{1}\right)}{4 \pi\left|\xi-\eta^{*}\right|^{2}}\left\{c(\xi)|\xi|^{2} \Psi_{1 \xi_{1}}(\xi) \Psi_{2}(\eta)-c(\eta)|\eta|^{2} \Psi_{1 \eta_{1}}(\eta) \Psi_{2}(\xi)\right\} \\
V I I I & =\frac{\left(\xi_{2}+\eta_{2}\right)}{2 \pi\left|\xi-\eta^{*}\right|^{2}}\left\{c(\xi) \xi_{2} \Psi_{1}(\xi) \Psi_{2}(\eta)+c(\eta) \eta_{2} \Psi_{1}(\eta) \Psi_{2}(\xi)\right\} \\
I X & =\frac{\left(\xi_{2}+\eta_{2}\right)}{4 \pi\left|\xi-\eta^{*}\right|^{2}}\left\{c(\xi)|\xi|^{2} \Psi_{1 \xi_{2}}(\xi) \Psi_{2}(\eta)+c(\eta)|\eta|^{2} \Psi_{1 \eta_{2}}(\eta) \Psi_{2}(\xi)\right\} .
\end{aligned}
$$

Similarly to $G_{1}$, the estimate

$$
\begin{aligned}
& \left|V I+V I I-\frac{\left(\xi_{1}-\eta_{1}\right)^{2}}{2 \pi\left|\xi-\eta^{*}\right|^{2}} \Psi_{1}(\xi) \Psi_{2}(\eta)\right| \\
& \leq C R^{-1}(|x|+|y|) \psi_{1}(x)^{1 / 2} \psi_{2}(y)+C R^{-1}|y| \psi_{2}(y)
\end{aligned}
$$

holds. On the other hand, we have

$$
\begin{aligned}
V I I I= & \frac{\left(\xi_{2}+\eta_{2}\right)^{2}}{2 \pi\left|\xi-\eta^{*}\right|^{2}} c(\xi) \Psi_{1}(\xi) \Psi_{2}(\eta) \\
& -\frac{\left(\xi_{2}+\eta_{2}\right) \eta_{2}}{2 \pi\left|\xi-\eta^{*}\right|^{2}}\left\{(c(\xi)-c(\eta)) \Psi_{1}(\xi) \Psi_{2}(\eta)\right. \\
& \left.+c(\eta)\left(\Psi_{1}(\xi)-\Psi_{1}(\eta)\right) \Psi_{2}(\eta)+c(\eta) \Psi_{1}(\eta)\left(\Psi_{2}(\eta)-\Psi_{2}(\xi)\right)\right\} \\
= & \frac{\left(\xi_{2}+\eta_{2}\right)^{2}}{2 \pi\left|\xi-\eta^{*}\right|^{2}} c(\xi) \Psi_{1}(\xi) \Psi_{2}(\eta) \\
& +\frac{\left(\xi_{2}+\eta_{2}\right) \eta_{2}}{2 \pi\left|\xi-\eta^{*}\right|^{2}} O(|\xi-\eta|)\left(\Psi_{1}(\xi) \Psi_{2}(\eta)+O\left(R^{-1}\right) \Psi_{2}(\eta)\right. \\
& \left.\quad+O\left(R^{-1}\right) \Psi_{1}(\eta)\right) .
\end{aligned}
$$

Noting (3.3), $\operatorname{supp} \Psi_{i} \subset B_{32 R}(0), \xi_{2} \geq 0, \eta_{2} \geq 0$, and $\left|D^{\alpha} \Psi_{i}\right|=O\left(R^{-|\alpha|}\right)$, we get

$$
\begin{aligned}
\Psi_{1 \xi_{2}}(\xi)+\Psi_{1 \eta_{2}}(\eta) & =\xi_{2}\left(1+O\left(R^{-2}\right) O(|\xi|)\right)+\eta_{2}\left(1+O\left(R^{-2}\right) O(|\eta|)\right) \\
& =\left(\xi_{2}+\eta_{2}\right)\left(1+O\left(R^{-2}\right) O(|\xi|+|\eta|)\right) \\
& =O\left(R^{-1}\right)\left(\xi_{2}+\eta_{2}\right) .
\end{aligned}
$$

This implies

$$
\begin{aligned}
I X= & \frac{\left(\xi_{2}+\eta_{2}\right)}{4 \pi\left|\xi-\eta^{*}\right|^{2}}\left\{(c(\xi)-c(\eta))|\xi|^{2} \Psi_{1 \xi_{2}}(\xi) \Psi_{2}(\eta)\right. \\
& +c(\eta)\left(|\xi|^{2}-|\eta|^{2}\right) \Psi_{1 \xi_{2}}(\xi) \Psi_{2}(\eta)+c(\eta)|\eta|^{2}\left(\Psi_{1 \xi_{2}}(\xi)+\Psi_{1 \eta_{2}}(\eta)\right) \Psi_{2}(\eta) \\
& \left.-c(\eta)|\eta|^{2} \Psi_{1 \eta_{2}}(\eta)\left(\Psi_{2}(\eta)-\Psi_{2}(\xi)\right)\right\} \\
= & \frac{\left(\xi_{2}+\eta_{2}\right)}{4 \pi\left|\xi-\eta^{*}\right|^{2}}\left\{O(|\xi-\eta|)|\xi|^{2} \Psi_{1 \xi_{2}}(\xi) \Psi_{2}(\eta)\right. \\
& +c(\eta) O(|\xi-\eta|) O(|\xi|+|\eta|) \Psi_{1 \xi_{2}}(\xi) \Psi_{2}(\eta) \\
& +c(\eta)|\eta|^{2}\left(\xi_{2}+\eta_{2}\right) O\left(R^{-1}\right) \Psi_{2}(\eta) \\
& \left.+c(\eta)|\eta|^{2} \Psi_{1 \eta_{2}}(\eta) O\left(R^{-1}\right) O(|\xi-\eta|)\right\} \\
= & O\left(R^{-1}\right)|\xi|^{2} \Psi_{1}^{1 / 2}(\xi) \Psi_{2}(\eta)+O\left(R^{-1}\right) O(|\xi|+|\eta|) \Psi_{1}^{1 / 2}(\xi) \Psi_{2}(\eta) \\
& +O\left(R^{-1}\right)|\eta|^{2} \Psi_{2}(\eta)+O\left(R^{-2}\right)|\eta|^{2} \Psi_{1}^{1 / 2}(\eta)
\end{aligned}
$$


Those relations are summarized as

$$
\left|\rho_{2}(x, y)-\frac{1}{2 \pi} \psi_{1}(x) \psi_{2}(y)\right| \leq \frac{C}{R}(|x|+|y|) \psi_{1}(x)^{1 / 2} \psi_{2}(y)+\frac{C}{R}|y| \psi_{2}(y) .
$$

Finally, because $K$ is a $C^{1, \theta}$ function, we have

$$
\begin{aligned}
& \psi_{2}(y) \nabla\left(m \psi_{1}\right)(x) \cdot \nabla_{x} K(x, y)+\psi_{2}(y) \nabla\left(m \psi_{1}\right)(y) \cdot \nabla_{y} K(x, y) \\
& =\psi_{2}(y)\left(O(1)|x| \psi_{1}(x)+O\left(R^{-1}\right)|x|^{2} \psi_{1}^{1 / 2}(x)\right) \\
& \quad+\psi_{2}(x)\left(O(1)|y| \psi_{1}(y)+O\left(R^{-1}\right)|y|^{2} \psi_{1}^{1 / 2}(y)\right) \\
& =O(1)\left(|x| \psi_{1}(x) \psi_{2}(y)+|x| \psi_{1}(x)^{1 / 2} \psi_{2}(y)\right) \\
& \quad+O(1)\left(|y| \psi_{1}(y) \psi_{2}(x)+|y| \psi_{1}(y)^{1 / 2} \psi_{2}(x)\right)
\end{aligned}
$$

The proof is complete.

Now, we are able to give the following.

Proof of Theorem 3. Since $\frac{\partial X_{1}}{\partial \nu}=X_{2}=0$ on $\partial \Omega$, we have

$$
\begin{aligned}
\frac{\partial m}{\partial \nu} & =\nu \cdot\left(\frac{1}{2} \nabla_{\xi}|\xi|^{2} \frac{\partial X}{\partial x}\right)=\frac{\partial X}{\partial \nu} \cdot X \\
& =\frac{\partial X_{2}}{\partial \nu} \cdot X_{2}=0 \quad \text { on } \partial \Omega .
\end{aligned}
$$

Also (3.1) implies that $m(x)=\frac{1}{2}|x|^{2}+O\left(|x|^{3}\right), m_{x_{i}}=x_{i}+O\left(|x|^{2}\right)$, and $m_{x_{i} x_{j}}=$ $\delta_{i j}+O(|x|)$ as $|x| \rightarrow 0$.

Let

$$
I_{\psi_{i}}(t)=\int_{\Omega} u(x, t) m(x) \psi_{i}(x) d x .
$$

The first equation of (1.5) gives

$$
\begin{aligned}
\frac{d}{d t} I_{\psi_{1}} & =\int_{\Omega} u_{t} m \psi_{1} d x=-\int_{\Omega}(\nabla u-u \nabla v) \cdot \nabla\left(m \psi_{1}\right) d x \\
& =\int_{\Omega} u \triangle\left(m \psi_{1}\right) d x+\int_{\Omega} u \nabla v \cdot \nabla\left(m \psi_{1}\right) d x \\
& =I+I I .
\end{aligned}
$$

The inequalities

$$
\left|\nabla \psi_{i}\right| \leq C R^{-1} \psi_{i}^{1 / 2} \quad \text { and } \quad\left|\Delta \psi_{i}\right| \leq C R^{-2} \psi_{i}^{1 / 2}
$$

hold for $\psi_{i}=\phi_{i}^{4}$. We obtain

$$
\begin{aligned}
I & =\int_{\Omega} u\left\{\psi_{1} \Delta m+2 \nabla m \cdot \nabla \psi_{1}+m \triangle \psi_{1}\right\} d x \\
& \leq 2 \int_{\Omega} u \psi_{1} d x+C R^{-1} \int_{\Omega}|x| \psi_{1}^{1 / 2} u d x \\
& \leq 2 \lambda_{\psi_{1}}+C R^{-1} \lambda I_{\psi_{1}}^{1 / 2} .
\end{aligned}
$$


The second equation of (1.5) implies

$$
\begin{aligned}
I I= & \int_{\Omega} \int_{\Omega} u(x, t) \nabla_{x} G(x, y) \cdot \nabla_{x}\left(m \psi_{1}\right)(x) u(y, t) d x d y \\
= & \int_{\Omega} \int_{\Omega} u(x, t) \psi_{2}(y) \nabla_{x} G(x, y) \cdot \nabla_{x}\left(m \psi_{1}\right)(x) u(y, t) d x d y \\
& +\int_{\Omega} \int_{\Omega} u(x, t)\left(1-\psi_{2}(y)\right) \nabla_{x} G(x, y) \cdot \nabla_{x}\left(m \psi_{1}\right)(x) u(y, t) d x d y \\
= & I I I+I V .
\end{aligned}
$$

Here, we have

$$
\operatorname{dist}\left(\operatorname{supp}\left(1-\psi_{2}\right), \operatorname{supp} \psi_{1}\right) \geq \operatorname{dist}\left(\mathbf{R}^{2} \backslash B_{16 R}(0), B_{8 R}(0)\right)=8 R
$$

and hence

$$
\begin{aligned}
I V & \leq C R^{-1} \int_{\Omega} \int_{\Omega}|x| \psi_{1}^{1 / 2}(x) u(x, t) u(y, t) d x d y \\
& \leq C R^{-1} \lambda \int_{\Omega}|x| \psi_{1}(x)^{1 / 2} u(x, t) d x \\
& \leq C R^{-1} \lambda^{3 / 2} I_{\psi_{1}}^{1 / 2}
\end{aligned}
$$

follows. On the other hand, we have

$$
I I I=\frac{1}{2} \int_{\Omega} \int_{\Omega} u(x, t) \rho(x, y) u(y, t) d x d y,
$$

and Lemma 3.2 implies

$$
\begin{aligned}
& \left|I I I-\frac{1}{2 \pi} \lambda_{\psi_{1}} \lambda_{\psi_{2}}\right| \\
& \leq \int_{\Omega} \int_{\Omega} u(x, t) \frac{1}{2}\left|\rho(x, y)-\frac{1}{\pi} \psi_{1}(x) \psi_{2}(y)\right| u(y, t) d x d y \\
& \leq C R^{-1} \lambda\left\{\int_{\Omega}|x| \psi_{1}^{1 / 2}(x) u(x, t) d x+\int_{\Omega}|y| \psi_{2}(y) u(y, t) d x\right\} \\
& =C R^{-1} \lambda \int_{\Omega}|x|\left(\psi_{1}(x)^{1 / 2}+\psi_{1}(x)\right) u(x, t) d x \\
& \quad+C R^{-1} \lambda \int_{\Omega}|x|\left(\psi_{2}(x)-\psi_{1}(x)\right) u(x, t) d x \\
& \leq C R^{-1} \lambda^{3 / 2} I_{\psi_{1}}^{1 / 2}+C \lambda \int_{\Omega}\left(\psi_{2}(x)-\psi_{1}(x)\right) u(x, t) d x .
\end{aligned}
$$

Consequently, we obtain

$$
I I I \leq \frac{1}{2 \pi} \lambda_{\psi_{1}}^{2}+C R^{-1} \lambda^{3 / 2} I_{\psi_{1}}^{1 / 2}+C \lambda \int_{\Omega}\left(\psi_{2}(x)-\psi_{1}(x)\right) u(x, t) d x .
$$

Those relations are summarized as

$$
\frac{d}{d t} I_{\psi_{1}} \leq 2 \lambda_{\psi_{1}}-\frac{1}{2 \pi} \lambda_{\psi_{1}}^{2}+C_{*} R^{-1} \lambda^{3 / 2} I_{\psi_{1}}^{1 / 2}+C_{*} \lambda\left(\lambda_{\psi_{2}}-\lambda_{\psi_{1}}\right)
$$


for $t \in\left[0, T_{\max }\right)$ with a constant $C_{*}>0$ independent of $R$.

By Lemma 3.1, there exists a constant $L>0$ satisfying

$$
\left|\frac{d}{d t} \lambda_{\psi_{i}}\right| \leq L R^{-2}\left(\lambda^{2}+\lambda\right)
$$

for $i=1,2$. Letting $\delta=\lambda_{\psi_{1}}(0)-4 \pi>0$, we take $T_{1}>0$ in

$$
L T_{1}\left(\lambda^{2}+\lambda\right) R^{-2}+C_{*} L T_{1}\left(\lambda^{3}+\lambda^{2}\right) R^{-2}<\delta / 4 .
$$

This implies

$$
\left|\lambda_{\psi_{i}}(t)-\lambda_{\psi_{i}}(0)\right|+C_{*} \lambda\left|\lambda_{\psi_{i}}(t)-\lambda_{\psi_{i}}(0)\right|<\frac{\delta}{4}
$$

in particular, where $t \in\left[0, \min \left(T_{1}, T_{\max }\right)\right)$.

We suppose that $\eta>0$ is so small that

$$
I_{\psi_{1}}(0)<\frac{\delta T_{1}}{2}
$$

and

$$
C_{*} R^{-1} \lambda^{3 / 2} I_{\psi_{2}}^{1 / 2}(0)+\frac{1}{16} C_{*} R^{-2} \lambda I_{\psi_{2}}(0)<\frac{\delta}{2}
$$

hold. In this case we have

$$
\begin{aligned}
& C_{*} R^{-1} \lambda^{3 / 2} I_{\psi_{1}}(0)^{1 / 2}+C_{*} \lambda\left(\lambda_{\psi_{2}}(0)-\lambda_{\psi_{1}}(0)\right) \\
& \quad \leq C_{*} R^{-1} \lambda^{3 / 2} I_{\psi_{2}}(0)^{1 / 2}+\frac{1}{16} C_{*} R^{-2} \lambda I_{\psi_{2}}(0)<\frac{\delta}{2} .
\end{aligned}
$$

The right-hand side of (3.4) at $t=0$ is less than

$$
-2(4 \pi+\delta) \frac{\delta}{4 \pi}+\frac{\delta}{2}<-\frac{3 \delta}{2}
$$

For $t>0$ sufficiently small, $I_{\psi_{1}}(t)$ is monotone decreasing.

We suppose $T_{\max }=+\infty$ and derive a contradiction. For this purpose, first we show that $I_{\psi_{1}}$ is monotone decreasing on $\left[0, T_{1}\right]$. In fact, otherwise we have $T_{0} \in\left(0, T_{1}\right)$ satisfying

$$
\frac{d}{d t} I_{\psi_{1}}\left(T_{0}\right)=0
$$

with $I_{\psi_{1}}$ being monotone decreasing on $\left[0, T_{0}\right]$. In use of (3.5) with $\lambda_{\psi_{1}}(0)>4 \pi$, we see that the right-hand side of (3.4) at $t=T_{0}$ is less than

$$
\begin{aligned}
& 2\left(\lambda_{\psi_{1}}(0)-\frac{\delta}{4}\right)-\frac{1}{2 \pi}\left(\lambda_{\psi_{1}}(0)-\frac{\delta}{4}\right)^{2}+C_{*} R^{-1} \lambda^{3 / 2} I_{\psi_{1}}(0)^{1 / 2} \\
& \quad+C_{*} \lambda\left(\lambda_{\psi_{2}}(0)-\lambda_{\psi_{1}}(0)\right) \\
& \quad+C_{*} \lambda\left(\left|\lambda_{\psi_{1}}\left(T_{0}\right)-\lambda_{\psi_{1}}(0)\right|+\left|\lambda_{\psi_{2}}\left(T_{0}\right)-\lambda_{\psi_{2}}(0)\right|\right) \\
& \leq-\frac{3 \delta}{8 \pi}\left(4 \pi+\frac{3 \delta}{4}\right)+C_{*} R^{-1} \lambda^{3 / 2} I_{\psi_{1}}(0)^{1 / 2}+C_{*} \lambda\left(\lambda_{\psi_{2}}(0)-\lambda_{\psi_{1}}(0)\right)+\frac{\delta}{2} .
\end{aligned}
$$


This implies

$$
\frac{d}{d t} I_{\psi_{1}}\left(T_{0}\right)<-\frac{\delta}{2}
$$

by (3.7), a contradiction.

At the same time we have proven that

$$
\frac{d}{d t} I_{\psi_{1}}(t)<-\frac{\delta}{2} \quad\left(t \in\left[0, T_{1}\right)\right)
$$

Therefore, (3.6) implies $I_{\psi_{1}}\left(T_{1}\right)<0$. This contradicts the positivity of the solution, and hence $T_{\max }<+\infty$ has been proven. The proof is complete.

The case $x_{0} \in \Omega$ is treated similarly. If

$$
\int_{B_{R}\left(x_{0}\right)} u_{0}(x) d x>8 \pi \quad \text { and } \quad \frac{1}{R^{2}} \int_{B_{R}\left(x_{0}\right)} u_{0}(x)\left|x-x_{0}\right|^{2} d x<\eta
$$

holds for sufficiently small $R>0$, then $T_{\max }<+\infty$ follows. Here, $\eta>0$ is a constant determined by $\lambda=\left\|u_{0}\right\|_{1}$ and $\left\|u_{0}\right\|_{L^{1}\left(B_{R}\left(x_{0}\right)\right)}$.

4. Blowup solution in the infinite time. This section is devoted to the proof of Theorem 2. We study (1.5) for $\Omega=\left\{x \in \mathbf{R}^{2}|| x \mid<1\right\}$. We set $a=1$ for simplicity and suppose (1.10) with $v_{0}=(-\Delta+1)^{-1} u_{0}$ and $T_{\max }=+\infty$. The initial value $u_{0}$ and hence the solution $u(t)$ are radially symmetric.

In [15], the relations

$$
\sup _{t \in[0,+\infty)}\|u(t)\|_{L^{\infty}\left(\Omega \backslash B_{R}(0)\right)}<+\infty
$$

and

$$
\sup _{t \in[0,+\infty)}\|v(t)\|_{L^{\infty}\left(\Omega \backslash B_{R}(0)\right)}<+\infty
$$

are shown for this case, where $R \in(0,1)$ is arbitrary. From the standard elliptic and parabolic estimates, this implies

$$
\|u\|_{C^{2,1}\left(\left(\Omega \backslash B_{R}(0)\right) \times[0,+\infty)\right)}<+\infty \quad \text { and } \quad\|v\|_{C^{2,1}\left(\left(\Omega \backslash B_{R}(0)\right) \times[0,+\infty)\right)}<+\infty .
$$

The following inequality is proven similarly to Lemma 13 of [9]. We have only to make use of the elliptic estimate instead of Lemma 12 there: Let $w$ be a solution to

$$
\begin{aligned}
-\Delta w+w & =f \text { in } \Omega \\
\frac{\partial w}{\partial \nu} & =0 \text { on } \partial \Omega .
\end{aligned}
$$

Then, it holds that

$$
\int_{B_{R}(0)} e^{w} d x \leq e^{C\|w\|_{L^{p}(\Omega)}} \cdot \int_{B_{2 R}(0)}\left(\frac{2}{|x|}\right)^{\theta} d x
$$

for $p>1$ and $\theta=\left\|f^{+}\right\|_{L^{1}\left(B_{2 R}(0)\right)} /(2 \pi)$.

Now we give the following. 
Proof of Theorem 2. As is noted at the end of $\S 2$, we have (2.3) with $\alpha>1 / 2$ in this case. Because of (4.1) this implies that

$$
\lim _{t \nearrow+\infty} \int_{B_{R}(0)} e^{\alpha v(t)} d x=+\infty
$$

for $R \in(0,1)$. We also have $\|v(t)\|_{W^{1, q}(\Omega)}=O(1)$ for $q \in[1,2)$ from the second equation of (1.5) in use of $\|u(t)\|_{1}=\lambda$ and the $L^{1}$ estimate ([5]). This implies

$$
\liminf _{t \nearrow+\infty} \int_{B_{R}(0)} u d x \geq 8 \pi
$$

from the above estimate on $w=\alpha v$. We have only to show that

$$
\lim _{R \searrow 0} \limsup _{t \nearrow+\infty} \int_{B_{R}(0)} u d x \leq 8 \pi .
$$

If this is not the case, we have

$$
\lim _{R \searrow 0} \limsup _{t \nearrow+\infty} \int_{B_{R}(0)} u d x>8 \pi .
$$

There exists $t_{k} \rightarrow+\infty$ and $\delta>0$ such that

$$
\int_{B_{R}(0)} u\left(t_{k}\right) d x>8 \pi+\delta
$$

for $R \in(0,1]$. Passing through a subsequence, we get a non-negative radially symmetric function $f \in L^{1}(\Omega) \cap C(\bar{\Omega} \backslash\{0\})$ satisfying

$$
u\left(t_{k}\right) \rightarrow f \quad \text { in } \quad L_{l o c}^{q}(\bar{\Omega} \backslash\{0\})
$$

for $q \geq 1$. Taking $\varepsilon \in(0, R)$, we have

$$
\begin{aligned}
& \limsup _{k \rightarrow \infty} \int_{B_{R}(0)} u\left(x, t_{k}\right)|x|^{2} d x \\
& \leq \limsup _{k \rightarrow \infty} \int_{B_{\varepsilon}(0)} u\left(x, t_{k}\right)|x|^{2} d x+\limsup _{k \rightarrow \infty} \int_{B_{R}(0) \backslash B_{\varepsilon}(0)} u\left(x, t_{k}\right)|x|^{2} d x \\
& \leq \lambda \varepsilon^{2}+R^{2} \int_{B_{R}(0)} f(x) d x .
\end{aligned}
$$

This implies

$$
\limsup _{k \rightarrow \infty} \int_{B_{R}(0)} u\left(x, t_{k}\right)|x|^{2} \leq R^{2} \int_{B_{R}(0)} f(x) d x .
$$

Because of $f \in L^{1}(\Omega)$, any $\eta>0$ admits $R \in(0,1]$ and an integer $k$ satisfying

$$
\frac{1}{R^{2}} \int_{B_{R}(0)} u\left(x, t_{k}\right)|x|^{2} d x<\eta
$$

However, as is noted at the end of $\S 3$, those relations imply $T_{\max }<+\infty$, a contradictioin. We have (4.2) and the proof is complete. 


\section{REFERENCES}

[1] Alikakos, N.D., $L^{p}$ bounds of solutions of reaction diffusion equations, Comm. Partial Differential Equations, 4 (1979), pp. 827-868.

[2] ALt, W., Biased random walk models for chemotaxis and related diffusion approximations, J. Math. Biol., 9 (1980), pp. 147-177.

[3] BILER, P., Local and global solvability of some parabolic systems modelling chemotaxis, Adv. Math. Sci. Appl., 8 (1998), pp. 715-743.

[4] Brezis, H., MERLE, F., Uniform estimates and blow-up behavior for solutions of $-\Delta u=$ $V(x) e^{u}$ in two dimensions, Comm. Partial Differential Equations, 16 (1991), pp. 12231253.

[5] Brezis, H. Strauss, W.A., Semi-linear second-order elliptic equations in $L^{1}$, J. Math. Soc. Japan, 25 (1973), pp. 565-590.

[6] Chang, S.Y.A., YAng, P.C., Conformal deformation of metrics on $S^{2}$, J. Differential Geometry, 27 (1988), pp. 259-296.

[7] Childress, S., Percus, J.K., Nonlinear aspects of chemotaxis, Math. Biosci., 56 (1981), pp. $217-237$.

[8] GajewsKi, H., ZAChARIAS, K., Global behaviour of a reaction-diffusion system modelling chemotaxis, Math. Nachr., 195 (1998), pp. 77-114.

[9] Harada, G., Nagai, T., Senba, T., Suzuki, T., Concentration lemma, Brezis-Merle type inequality, and a parabolic system of chemotaxis, Advances in Differential Equations, 6 (2001), pp. 1255-1280.

[10] Herrero, M.A., Velázquez, J.J.L., Singularity patterns in a chemotaxis model, Math. Ann., 306 (1996), pp. 583-623.

[11] Herrero, M.A., Velázquez, J.J.L., A blow-up mechanism for a chemotaxis model, Ann. Scuola Norm. Sup. Pisa IV, 35 (1997), pp. 633-683.

[12] Horstmann, D., WANG, G., Blowup in a chemotaxis model without symmetry assumptions, Euro. Jnl. of Applied Mathematics, 12 (2001), pp. 159-177.

[13] JÄGER, W., LuCKHAUS, S., On explosions of solutions to a system of partial differential equations modelling chemotaxis, Trans. Amer. Math. Soc., 329 (1992), pp. 819-824.

[14] Keller, E.F., SEgel, L.A., Initiation of slime mold aggregation viewed as an instability, J. Theor. Biol., 26 (1970), pp. 399-415.

[15] NAGAI, T., Blow-up of radially symmetric solutions to a chemotaxis system, Adv. Math. Sci. Appl., 5 (1995), pp. 581-601.

[16] NAGAI, T., Blowup of nonradial solutions to parabolic - elliptic systems modeling chemotaxis in two-dimensional domains, J. Inequal. Appl., 6 (2001), pp. 37-55.

[17] Nagai, T., Senba, T., Suzuki, T., Chemotactic collapse in a parabolic system of chemotaxis, Hiroshima Math. J., 30 (2000), pp. 463-497.

[18] Nagai, T., Senba, T., Yoshida, K., Application of the Trudinger-Moser inequality to a parabolic system of chemotaxis, Funkcial. Ekvac., 40 (1997), pp. 411-433.

[19] NAGASAKI, K., SUZUKI, T., Asymptotic analysis for two-dimensional elliptic eigenvalue problems with exponentially-dominated nonlinearities, Asymptotic Analysis, 3 (1990), pp. 173188.

[20] Nanjundiah, V., Chemotaxis, signal relaying, and aggregation morphology, J. Theor. Biol., 42 (1973), pp. 63-105.

[21] SEnBA, T., SuZUKI, T., Some structures of the solution set for a stationary system of chemotaxis, Adv. Math. Sci. Appl., 10 (2000), pp. 191-224.

[22] Senba T., SuzuKI, T., Chemotactic collapse in a parabolic - elliptic system of mathematical biology, Advances in Differential Equations, 6 (2001), pp. 21-50.

[23] Senba, T., SuzuKi, T., Local and norm behavior of blow up solutions to a parabolic system of chemotaxis, J. Korean Math. Soc., 37 (2000), pp. 929-942.

[24] SUzUKI, T., Global analysis for a two-dimensional elliptic eigenvalue problem with the exponential nonlinearity, Ann. Inst. H. Poincaré, Analyse nonlinéaire, 9 (1992), pp. 367-398.

[25] SUZUKI, T., A note on the stability of stationary solutions to a parabolic system of chemotaxis, Communications in Contemporary Mathematics, 2 (2000), pp. 407-428.

[26] YAGI, A., Norm behavior of solutions to the parabolic system of chemotaxis, Math. Japonica, 45 (1997), pp. 241-265. 
T. SENBA AND T. SUZUKI 